\title{
LA TOPONIMIA DE LOS ANTIGUOS REINOS ARIOS
}

\author{
José Luis Blesa Cuenca \\ (Universidad Autónoma de Madrid)
}

\begin{abstract}
RESUMEN
El presente artículo pretende situar geográficamente los topónimos de los reinos arios de la temprana Edad del Hierro recogidos en las fuentes antiguas y relacionarlos con las diferentes manifestaciones regionales de su cultura material, trazando un mapa en el que se distingan los diversos reinos y se apunten cuáles serían sus ciudades principales entre el conjunto de yacimientos catalogados.
\end{abstract}

\section{PALABRAS CLAVE}

Arios, cerámica modelada pintada, satrapias, toponimia, Asia Central.

\section{THE TOPONYMY OF THE ANCIENT ARYAN KINGDOMS}

\begin{abstract}
This paper aims to locate geographically the toponyms of the Early Iron Age Aryan kingdoms collected in the ancient sources and relate them to the different regional manifestations of their material culture. With this material a map will be drawn in which the different kingdoms and which would be their main cities are pointed out among the set of cataloged sites.
\end{abstract}

\section{KEYWORDS}

Aryans, hand-made painted pottery, satrapies, Toponymy, Central Asia.

\section{1- Introducción}

Han pasado casi cuarenta años desde que Karlheinz Kessler publicase su célebre "Untersuchungen zur historischen Topographie Nordmesopotamiens nach keilschriftlichen

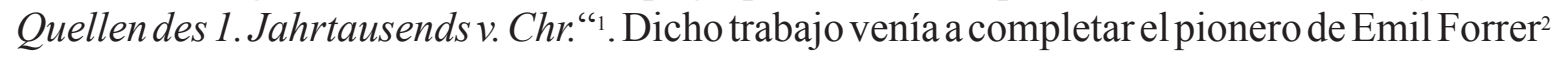
sobre la división provincial asiria, y en cierta medida también el Neo-Assyrian toponyms, que una década antes había publicado Simo Parpola ${ }^{3}$. Desde entonces, se convertiría en una obra de obligada consulta para cualquier estudio que implicase una referencia a la toponimia del imperio asirio, y así lo sigue siendo, pese a las pequeñas matizaciones que, pasado casi medio siglo, se han ido haciendo y fruto de las cuales resulta el trabajo de Michael Porter y Simo Parpola en The Helsinki atlas of the Near East in the Neo-Assyrian period ${ }^{4}$.

Pese a todo, la seguridad con la que se sitúan los topónimos se va haciendo más frágil conforme nos alejamos hacia el Este. Las referencias en las fuentes cuneiformes ${ }^{5}$ son escasas y a menudo imprecisas, pues raramente los asirios se aventuraban directamente en lugares tan alejados como Partia, aunque los contactos, sin duda, existieron. Por ello los topónimos más orientales, tales como "el país de los diauehi"; "el país de la casa de la sal"; el monte Bikni, "la montaña de lapislázuli"; y "el país de los medos lejanos”, donde se encontraban

\footnotetext{
${ }^{1}$ K. Kessler.- Untersuchungen zur historischen Topographie Nordmesopotamiens nach keilschriftlichen Quellen des 1. Jahrtausends v. Chr. Wiesbaden 1980. Trabajo que se enmarcaba en la serie de trabajos del Tübinger Atlas des Vorderens Orients, donde en 1973 Wolfgang Röllig había editado las láminas correspondientes a la Edad del Hierro (B-IV).

${ }^{2}$ E. Forrer.- Die Provinzeinteilung des assyrischen Reiches, Leipzig 1920.

${ }^{3}$ S. Parpola.- Neo-Assyrian toponyms, Kevelaer 1970.

${ }^{4}$ M. Porter y S. Parpola.- The Helsinki atlas of the Near East in the Neo-Assyrian period, Helsinki 2001.

5 J. L. Blesa Cuenca.- Partakka-Parthava-Partia. Los reinos preaqueménidas de Asia Central en las fuentes escritas neoasirias, Madrid 2013. (Trabajo de Fin de Máster sin publicar, defendido en la U. A. M. en 2013).
} 
Partakka, Partukka, Urakazabarna; se situaban en los trabajos arriba mencionado de una forma algo vaga, posición que hemos tenido la oportunidad de precisar al estudiar las referencias específicas que asirios, babilonios y urarteos hicieron a esos territorios y sus gentes, poniéndolo en relación con la evidencia arqueológica allí presente.

Pero de más allá del piedemonte del Kopet Dagh, ni siquiera los asirios han dejado testimonio. Para los territorios al oriente de Partia hemos de buscar referencias en otro tipo de fuentes, como son los textos avésticos y, para el caso de los reinos de India y Pakistán; los textos védicos.

\section{2- La organización político-territorial de los antiguos arios}

El Avesta ${ }^{6}$ describe en sus partes más antiguas una sociedad de pastores nómadas, que basaban su poder en el ganado bovino y ovicaprino y en la destreza de sus jinetes, la misma que encontramos en los yacimientos de la cultura de Andronovo. Sabemos que estos primeros pueblos arios se agruparían según modelos gentilicios en casa, aldea o clan -depende de cómo se traduzca-, tribu y país. Términos que son continuamente repetidos, y siempre en el mismo orden, como nmānō, vīspa, zaṇtu, daińhu en los textos avésticos (Y. 8, 7; 23, 1; 57, 14; 68, 5; 71,22 y sobre todo en los más antiguos textos Y. 31, 16; 31, 18). Cada una de estas divisiones sociales y políticas estaría bajo la autoridad de un señor (paiti), que serían el nmānō-paiti, vīspaiti, zaṇtupaiti y daińhupaiti respectivamente (Y. 9, 27; 13, 1; 19, 18). El daińhupaiti era la autoridad superior en el país, y hemos de suponer que sería el título que ostentaría Kava Vištāspa, el rey que acoge a Zaratustra bajo su protección cuando éste huye al no tener éxito predicando entre su pueblo, jinetes nómadas de la estepa que vivían en los márgenes de las sociedades urbanas, aunque por desgracia no tenemos ninguna fuente en la que se nos dé el título de este personaje.

Sí que resulta interesante para nosotros otro título, en este caso aplicado al dios Mitra, como señor de los países (dahyunąm daińhupaitīm) (Y. 3, 13; 4, 16; 6, 10; 7, 12.), pues nos indica que ya existía la idea de una autoridad -moral y divina, en este caso- superior al país y en consonancia con la idea de comunidad cultural, lo cual explicará la exitosa unión en bajo el caudillaje de Fraortes (672-669). Pero hasta su unión como imperio, lo que tenemos son estos reinos o países, los dahyō, que se organizan desde la capital, una ciudad donde reside el daińhupaiti y desde donde controla las actividades económicas que sostienen su reino; la ganadería y el control de las rutas caravaneras. Este modo de vida va a condicionar la morfología de los asentamientos, que van a estar dominados por una ciudad alta, a veces incluso fortificada como ciudadela, desde la que poder controlar, en medio de las vastas llanuras centroasiáticas, las rutas por las que circulaban los rebaños, las caravanas y los ejércitos.

\section{3- La toponimia de los reinos arios}

Además de coincidir la imagen que tenemos de todos estos reyes en cuanto a su modo de gobierno, sus actuaciones, y sus nombres de origen ario, la Arqueología, muestra una imagen uniforme de todo el territorio de los pueblos arios, como ha demostrado Johanna Lhuillier ${ }^{7}$

\footnotetext{
${ }^{6}$ Tómese como referencia la edición clásica de K. F. Geldner.- Avesta, the Sacred Books of the Parsis, Vol. I: Prolegomena Yasna, Stuttgart 1889. (reimpreso en Delhi en 1991); K. F. Geldner. Avesta, the Sacred Books of the Parsis, Vol. II: Vispered and Khorda Avesta, Stuttgart 1889. (reimpreso en Delhi en 1991); K. F. Geldner.Avesta, the Sacred Books of the Parsis, Vol. III: Vendidad, Stuttgart 1896. (reimpreso en Delhi en 1991). Para el Avesta antiguo, concretamente los Gathas; véase también la edición de Christian Bartholomae, completado por su discípulo Fritz Wolf; C. Bartholomae.- Die Gatha 's des Awesta, Zarathushtra 's Verspredigten, Strassburg 1905.; F. Wolff.- Avesta, die heiligen Bücher der Parsen, Leipzig 1910. (Reimpreso en Berlín en 1960).

${ }^{7}$ J. Lhuillier.- Le phénomène des ,, cultures à céramique modelée peinte ,, en Asie centrale dans l'évolution et la
} 
para el caso de la cerámica modelada pintada. El caso de la cerámica es un excelente ejemplo, puesto que aunque se observan perfectamente unos rasgos comunes que permiten identificarla como tal, el regionalismo en los diferentes lugares donde se documenta es claro, y coincide bastante bien con la división en satrapías que conocemos para época aqueménida, la cual sin duda se debió hacer sobre la base de los reinos ya existentes en esta época, entre los que no sólo encontramos las diferentes regiones de la cerámica modelada pintada (Partia, Margiana, Bactria y Sogdiana), sino también otras culturas similares pero con una cerámica algo distinta, como la del Dahistán Arcaico (Hircania), la cerámica del Hierro iraní (Rayy) o la cerámica gris pintada del noroeste de la India (Aria, Aracosia y Hapta hindu).

El dónde situamos exactamente dichos territorios sobre un mapa está determinado por las descripciones que de ellos tenemos en las fuentes clásicas ${ }^{8}$. El primer mapa que tenemos referencia es el de Aristágoras ( $c a$. 611-546) y poco después, el de Anaximandro (ca. 500)9 a los que les siguen el de Hecateo de Mileto $^{10}$, Heródoto ${ }^{11}$, Dicearco ${ }^{12}$ y Eratostenes. Y ya en época romana, el de Estrabón ${ }^{13}$. Sin embargo, ninguno de estos mapas se ha conservado. Es de especial interés para nosotros observar, cómo, en la Tabula peutingeriana, un mapa del s. XII o inicios del XIII, pero realizado a partir de uno romano del siglo IV, a la región formada por "Persida" (Persia), "Media maior" y "Parria" (Partia) se le llama en conjunto "Ariae". Evidencia de que se mantiene la concepción de todos estos países como pertenecientes a una misma cultura; la de los pueblos arios.

Sin embargo la culminación de la cartografía helenística, según la entendió Oswald A. W. Dilke $^{14}$ son los célebres mapas de Ptolomeo ${ }^{15}$ (1990), que tampoco han llegado hasta nosotros, pero sí fueron reconstruidos a partir de los textos o parcialmente derivados de los originales en los códices griegos que llegaron a Europa con los cruzados (ss. XII-XIII). La primera traducción

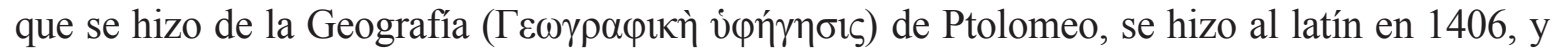
en los años siguientes se completó con la aparición de nuevas copias manuscritas; aunque su difusión se multiplicó con la primera edición impresa (Bolonia, 1477) ${ }^{16}$, a la que siguieron muchas otras traducciones al latín y a las lenguas vernáculas, sobre todo en Italia y Alemania. De estos mapas partirán las reconstrucciones históricas de las que somos herederos, y sobre ellas se fueron situando los topónimos de las fuentes antiguas.

Pero la existencia de esta división territorial es anterior a la organización provincial aqueménida, y su correspondencia con los reinos arios la demuestra no sólo la evidencia arqueológica, con el regionalismo en sus expresiones materiales coincidente con las posteriores

transformation des sociétés de la fin de l'âge du Bronze et du début de l'âge du Fer (Ile-Ier millénaire avant n.è.). Une synthèse comparative et régionale de la culture matérielle, Paris 2010. (Tesis de doctorado de la Université Paris I Panthéon-Sorbonne); J. Lhullier,- "Les cultures «à céramique modelée peinte» en Asie Centrale: un aperçu de 1'assemblage céramique de la deuxième moitié du 2e millénaire av. n.è.”, Iranica Antiqua XLVIII, 2013, 103-146.

${ }^{8}$ J. L. Blesa Cuenca.- Los arios. Historia y modos de vida de los pueblos centroasiáticos de la Edad del Hierro, Madrid 2018, 31-42. (Tesis inédita de doctorado de la U. A. M.)

9 J. B. Harley y D. Woodward. (eds.)- The History of Cartography, vol 1, Chicago 1987, 132-135.

${ }^{10}$ E. H. Bunbury.- A History of Ancient Geography among the Greeks and Romans from the Earliest Ages till the Fall of the Roman Empire, New York 1959, 148.

${ }^{11}$ R. Wilkinson.- Atlas Classica, London 1802.

${ }^{12}$ A. Cortesão.- History of Portuguese Cartography, vol. I, Lisboa 1970, fig. 16.

${ }^{13}$ E. H. Bunbury.- A History of Ancient Geography among the Greeks and Romans from the Earliest Ages till the Fall of the Roman Empire, New York 1959, 238.

${ }^{14}$ O. A. W. Dilke.- "The Culmination of Greek Cartography in Ptolemy”, en J. B. Harley y D. Woodward (eds.).Op. cit., Chicago 1987, 177-200.

${ }^{15}$ Véase la edición con introducción de L. Pagani y traducción al inglés por S. Knight.- Claudii Ptolemae Cosmographia tabulae/Maps from Ptolemy's Geography, Wigston 1990.

${ }^{16}$ De la que se publicó una edición facsímil en Amsterdam en 1963. 
satrapías, sino también el hecho de que ya se nombren en la descripción del mundo que se hace en el Avesta (Vendidad, Fargard 1) que, como estudiamos con profundidad en otro trabajo ${ }^{17}$, podemos retrotraerlo según criterios filológicos hasta esta época.

En el primer fargard de Vendidad, se listan los países de Sughdha (suүða, en antiguo

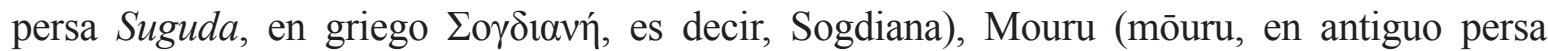

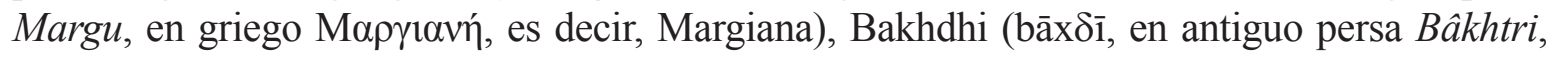
en griego $B \alpha A \eta \xi \xi i \alpha$, es decir, Bactria), Haroyu (harōyū, en antiguo persa Haraiva, en griego

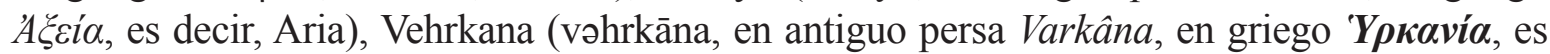
decir, Hircania), Harahvaiti (harax ${ }^{v}$ aitī, en antiguo persa Harauvati, en griego $A \xi \alpha \rho \omega \zeta i \alpha$, es decir, Aracosia), Ragha (rayą, en antiguo persa Ragâ, en griego Pó $\gamma \alpha \eta$, es decir, Rayy) o Hapta hindu (hapta həṇdu, en antiguo persa Hindava, en griego 'I $\lambda \delta v i$, es decir, India, referido aquí al Punjab). Llegados a este punto cabe mencionar que la existencia de Sogdiana como un reino independiente no es del todo segura. En época aqueménida no se conoce un sátrapa exclusivo de Sogdiana, sino que en los recientemente publicados documentos de Bactria se observa que la Sogdiana se gobernaba desde Bactria ${ }^{18}$. Khasanov ${ }^{19}$ ya había apuntado que durante la Edad del Hierro, por paralelos cerámicos en yacimientos sogdianos y bactrios, Sogdiana tenía que ser parte de Bactria. Quizá, como señalan Grenet y Rakhmanov²0, el centro de la Sogdiana preaqueménida habría sido Koktepe, desarrollándose Afrasiab más tarde, en época aqueménida.

Estos dahyō o mâtū, como los llamaban de forma más imprecisa los acadios, serían gobernados desde la capital por el daińhupaiti, al que debían fidelidad según un criterio de tipo cuasi feudal, gentilicio para ser más exactos, los zaṇtupaiti que estaban a la cabeza de las tribus. Son los bêlū âlānī o señores de las ciudades de las fuentes asirias, cuya común cultura, con una misma lengua, una misma mentalidad y unas mismas prácticas socioeconómicas, favorecieron su unión bajo un único mando, el de un caudillo o dahyunąm daińhupaitīm, para enfrentarse a las incursiones asirias.

\section{4- Conclusiones}

Gracias a toda esta información, hemos podido reconstruir la toponimia de los antiguos arios, entendiendo que la antigua civilización de Marhăši de las fuentes acadias se correspondía con lo que arqueológicamente se llamó el Complejo Arqueológico Bactro-Margiano ${ }^{21}$, y por lo tanto, en su ubicación, con la posterior Margiana. De la Partakka de tiempos de Assarhadon, que se trataba de la Parthawa persa, la que nosotros conocemos como Partia ${ }^{22}$. Y para los territorios orientales, tenemos las referencias avésticas arriba mencionadas. Tomando todos estos topónimos como referencia, y observando su coincidencia con el regionalismo en la cultura material apuntado por Johanna Lhuillier ${ }^{23}$ antes apuntado, hemos ido situando todos

\footnotetext{
${ }^{17}$ J. L. Blesa Cuenca.- Op. cit. Madrid 2018, 13-20.

${ }_{18}$ S. Shaked.- Le satrape de Bactriane et son gouverneur: documents arameens du IV s. avant notre ere provenant de Bactriane, Paris 2004.

${ }_{19}$ М. Х. Хасанов.- “Некоторые новые данные к становлению городской культуры в Согде”, en Т.Ш. Ширинов, у Ш.Р. Пидаев (eds.).- Роль города Самарканда в истории мирового культурного развития. Материаль Международного научного симпозиума, посвященного 2750-летнему юбилею города Самарканда, Ташкент/Самарканд 2007, 38-41.

${ }^{20}$ Ф. Гренье, у Ш. Рахманов.- “Новые данные о дате первой системы фортификации Афрасиаба.(середина или вторая половина VI в. до н.э.)”, еn Т.Ш. Ширинов, у Ш.Р. Пидаев (eds.).- Роль города Самарканда в истории мирового культурного развития.Материаль Международного научного симпозиума, посвященного 2750-летнему юбилею города Самарканда, Ташкент/Самарканд 2007, 23.

${ }^{21}$ H. P. Francfort y X. Tremblay.- "Marhaši et la civilisation de 1'Oxus", Iranica Antiqua 45, 2010, 51-224.

22 J. L. Blesa Cuenca.- Op. cit. Madrid 2013.

${ }^{23}$ J. Lhuillier.- Op. cit., Paris 2010; J. Lhullier,- Op. cit., 2013, 103-146.
} 
aquellos yacimientos con evidencias arqueológicas de los distintos pueblos arios, intentando -cuando el grado de desarrollo de las investigaciones lo permitiese-, distinguir cuáles fueron sus asentamientos principales. Fruto de esta investigación, resulta el presente mapa que proponemos, y cuya leyenda aquí detallamos:

(Con un cuadrado se señalan los principales asentamientos de cada reino, estando el resto de yacimientos marcados con un círculo rojo -en el caso de aquellos en los que las investigaciones han revelado una cierta entidad del asentamiento- o azul -en los que tan sólo se han identificado pequeñas manifestaciones de cultura material correspondiente a estas culturas, bien porque no se encontraron más o bien porque los trabajos arqueológicos han sido muy limitados)

1. Tabriz

2. Hasanlu

3. Tappe Qalayči (Izirtu)

4. Kul Tārike

5. Qal'e Ziwiye

6. Ardabil

7. Maryan

8. Tul

9. Vaske

10. Tales

11. Tepe Jalaliye

12. Jamshidabad/Marlik/Shahran

13. Ghias Abad/Ghale Kouti

14. Gardaneh Shar

15. Godin Tepe

16. Baba Jan

17. Nush-i Jan

18. Tappe Sangtarašan

19. Sorkdom-e Laki

20. Tepe Ozbaki

21. Vasun-e Kakhah

22. Zar Bolag

23. Tepe Qoli Darvish

24. Minas de Vešnave

25. Sarm

26. Shamshirgah

27. Tepe Sialk

28. Gandab-e Karand

29. Tepe Ma'morin

30. Gohar Tepe

31. Tureng Tepe

32. Benguvan

33. Izat Kuli

34. Madau depe

35. Geoktchik depe

36. Ovadan depe

37. Anau

38. Yashilly depe
39. Yassy depe

40. Dashly 17-30

41. El'ken depe

42. Tekkem depe

43. Garaoj depe

44. Ulug depe

45. Nishapur-P

46. Quchan

47. Tepe Hajji Bibi

48. Tepe Yam

49. Tepe Shirvan

50. Yaz depe

51. Uch depe

52. Aravali depe

53. Kushbegi depe

54. Dashly depe 1-6

55. Site n. 999

56. Otliyatan

57. Takhirbay 1

58. Takhirbay 3

59. Adam-Basan

60. Chopli depe

61. Taip

62. Odej depe

63. Dzham 53

64. Afrasiab

65. Koktepe

66. Erkurgan

67. Chirakchi

68. Turkul'tepa

69. Sangirtepe

70. Uzunkyr

71. Niezbashtepa

72. Kaunchitepe

73. Shahstepe

74. Kavardan

75. Tujabuguz 1-14

76. Khantepa 
77. Khozdzhent

78. Nurtepa

79. Ak-Tanga

80. Chust

81. Kara-kurgan

82. Tergauchi

83. Gurmiton

84. Tjura-kurgan

85. Tash-kurgan

86. Aktam

87. Akbarabad

88. Zarguldaktepe

89. Chakan

90. Dal'verzin

91. Ashkal-tepe

92. Akhshar/Gairat-tepe

93. Manjak

94. Dil'kusho

95. Osh

96. Djampalak

97. Chajan-tepe

98. Kosh-tepe

99. Bashbulak

100. Mady

101. Shaltak

102. Dolon-tepe

103. Koshok-tepe

104. Khozhambag

105. Kara-depe

106. Kurshab

107. Kyzyl Oktyabr

108. Boz-tepe

109. Dekhan

110. Dunbulak

111. Establecimiento 82

112. Establecimiento 33

113. Chimbaj

114. Karakochkor

115. Sartepe

116. Kuloltepe

117. Afghantepe

118. Kul'tepe

119. Kyzylcha 6

120. Kyzyl-tepe

121. Bujrachi-tepe

122. Bektepa

123. Majdatepa

124. Dzhanlavatepa
125. Dzharkutan

126. Kuchuk-tepe

127. Tashguzor

128. Teguzak

129. Kangurttut

130. Karim-Berdy

131. Naiabad/Shah tepe

132. Kumli

133. Imam-Sahib

134. Emshi-tepe

135. Tillya-tepe

136. Balambat

137. Timargarha

138. Ghalighai

139. Buktara

140. Aligrama

141. Zarif Karuna

142. Rupar

143. Sanghol

144. Bhagwanpura

145. Daulatpur

146. Raja-karan-ka-qila

147. Noh

148. Jodhpura

149. Ahichchhatra

150. Alamgirpur

151. Atranjikhera

152. Allahpur

153. Hulas

154. Hastinapur

155. Jakhera

156. Mathura

157. Sonkh

158. Kampil

159. Kurukshetra

160. Kotia

161. Pariar

162. Sravasti

163. Sringaverapura

164. Kausambi

165. Besnagar

166. Ujjain

167. Gilund

168. Sardargarh

169. Satavali

170. Harappa

171. Gharinda 


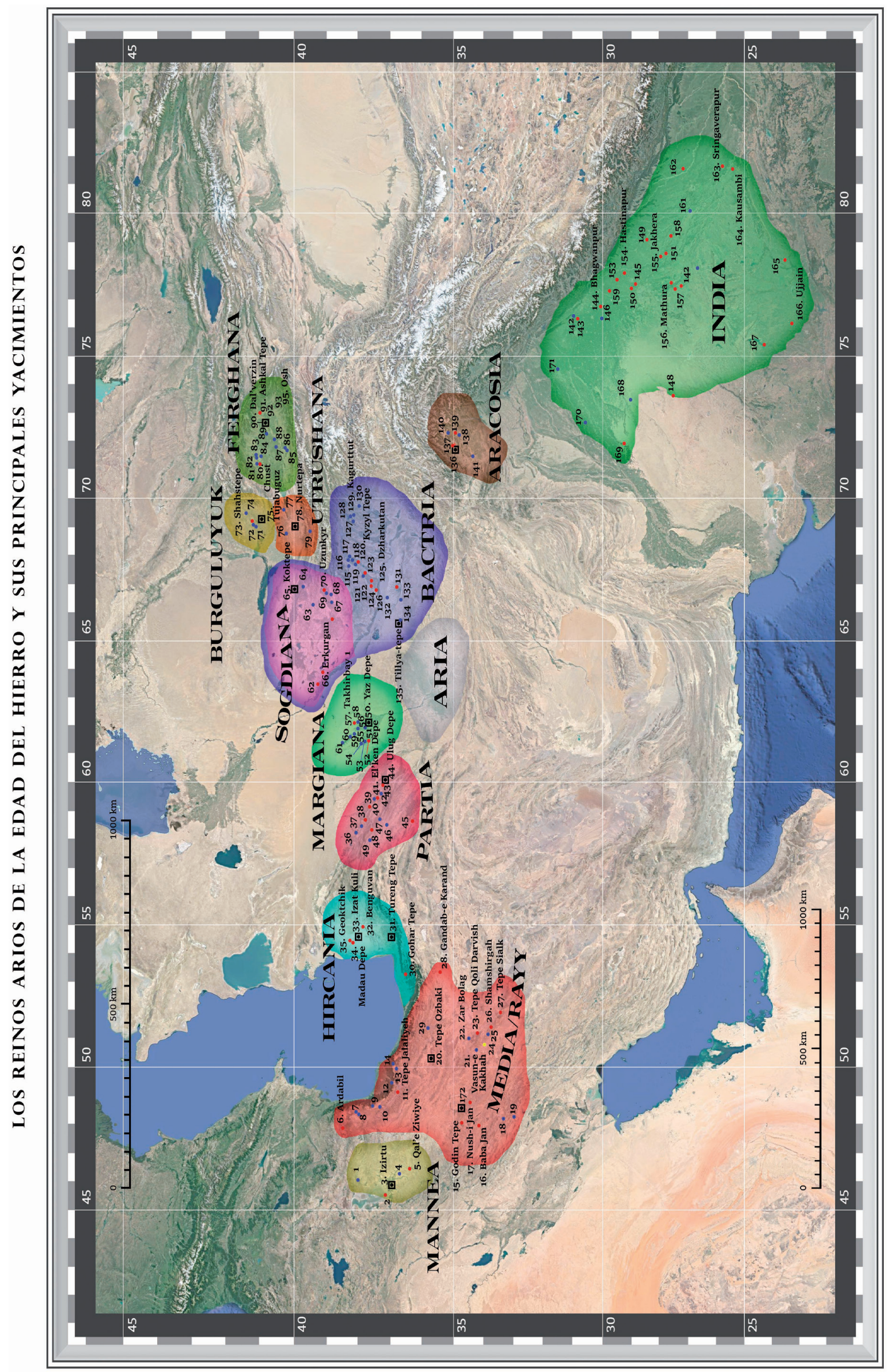




\section{BIBLIOGRAFÍA}

BARTHOLOMAE, C.

1905 Die Gatha's des Awesta, Zarathushtra's Verspredigten, Strassburg.

BLESA CUENCA, J. L.

2013 Partakka-Parthava-Partia. Los reinos preaqueménidas de Asia Central en las fuentes escritas neoasirias, Madrid. (Trabajo de Fin de Máster sin publicar, defendido en la U. A. M. en 2013).

2018 Los arios. Historia y modos de vida de los pueblos centroasiáticos de la Edad del Hierro, Madrid. (Tesis inédita de doctorado de la U. A. M.).

BUNBURY, E. H.

1959 A History of Ancient Geography among the Greeks and Romans from the Earliest Ages till the Fall of the Roman Empire, New York.

CORTESÃO, A.

1970 History of Portuguese Cartography, vol. I, Lisboa.

DILKE, O. A. W.

1987 “The Culmination of Greek Cartography in Ptolemy”, en J. B. Harley y D. Woodward (eds.).- Op. cit., Chicago, 177-200.

FORRER, E.

1920 Die Provinzeinteilung des assyrischen Reiches, Leipzig.

FRANCFORT, H. P. y TREMBLAY, X.

2010 "Marhaši et la civilisation de 1'Oxus", Iranica Antiqua 45, 51-224.

GELDNER, K. F.

1889 Avesta, the Sacred Books of the Parsis, Vol. I: Prolegomena Yasna, Stuttgart. (reimpreso en Delhi en 1991).

1889 Avesta, the Sacred Books of the Parsis, Vol. II: Vispered and Khorda Avesta, Stuttgart. (reimpreso en Delhi en 1991).

1896 Avesta, the Sacred Books of the Parsis, Vol. III: Vendidad, Stuttgart. (reimpreso en Delhi en 1991).

ГРЕНЬЕ, Ф. у РАХМАНОВ, Ш.

2007 “Новые данные о дате первой системы фортификации Афрасиаба.(середина или вторая половина VI в. до н.э.)”, еn Т.Ш. Ширинов, у Ш.Р. Пидаев (eds.).- Роль города Самарканда в истории мирового культурного развития.Материалы Международного научного симпозиума, посвященного 2750-летнему юбилею города Самарканда, Ташкент/Самарканд, 23.

HARLEY, J. B. y WOODWARD, D.

1987-1994 The History of Cartography, Chicago. 
XАCAHOB, M. X.

2007 “Некоторые новые данные к становлению городской культуры в Согде", en Т.Ш. Ширинов, у Ш.Р. Пидаев (eds.).- Роль города Самарканда в истории мирового культурного развития.Материаль Международного научного симпозиума, посвященного 2750-летнему юбилею города Самарканда, Ташкент/Самарканд, 38-41.

KESSLER, K.

1980 Untersuchungen zur historischen Topographie Nordmesopotamiens nach keilschriftlichen Quellen des 1. Jahrtausends v. Chr., Wiesbaden.

LHUILLIER, J.

1910 Le phénomène des „, cultures à céramique modelée peinte ,, en Asie centrale dans l'évolution et la transformation des sociétés de la fin de l'âge du Bronze et du début de l'âge du Fer (IIe-Ier millénaire avant n.è.). Une synthèse comparative et régionale de la culture matérielle, Paris. (Tesis de doctorado de la Université Paris I Panthéon-Sorbonne).

2013 "Les cultures «à céramique modelée peinte» en Asie Centrale: un aperçu de 1'assemblage céramique de la deuxième moitié du 2e millénaire av. n.è.", Iranica Antiqua XLVIII, 103-146.

PARPOLA, S.

1970 Neo-Assyrian toponyms, Kevelaer.

PORTER, M. y PARPOLA, S.

2001 The Helsinki atlas of the Near East in the Neo-Assyrian period, Helsinki.

PTOLOMEO, C.

1963 Cosmographia, Amsterdam. (edición facsimile de la impresa en Bolonia en 1477). 1990 Claudii Ptolemae Cosmographia tabulae/Maps from Ptolemy's Geography, Wigston. (introducción de L. Pagani y traducción de S. Knight).

SHAKED, S.

2004 Le satrape de Bactriane et son gouverneur: documents arameens du IV s. avant notre ere provenant de Bactriane, Paris.

SMITH, W.

1874 Atlas of Ancient Geography, London.

WILKINSON, R.

1802 Atlas Classica, London.

WOLFF, F.

1910 Avesta, die heiligen Bücher der Parsen, Leipzig. (Reimpreso en Berlín en 1960). 
